\title{
AGENDA 21 LOCAL COMO INSTRUMENTO DE ORDENACIÓN TERRITORIAL: LA MANCHA ALTA CONQUENSE
}

\author{
POR \\ JAVIER MARTÍNEZ VEGA* \\ Y MARÍA ASUNCIÓN MARTÍN LOU*
}

\section{Introducción}

La Agenda 21 Local es un documento que desarrolla un Plan Estratégico Municipal o Comarcal basado en la integración, con criterios sostenibles, de las políticas ambientales, económicas y sociales de la comarca y que surge de la participación y toma de decisiones consensuadas entre los representantes políticos, técnicos municipales, agentes implicados y ciudadanos de los territorios afectados. La Agenda 21 Local es el instrumento de gestión, de carácter no vinculante, más ampliamente aceptado por las autoridades locales para abordar los problemas ambientales, de forma participativa, desde el enfoque de «pensar globalmente y actuar localmente». En resumen, persigue integrar una justicia social, una economía sostenible y un medio ambiente duradero para lograr un equilibrio sostenible que se traduzca en una mejora de la calidad de vida.

Este instrumento de gestión surge del Programa Global para el Desarrollo Sostenible en el siglo XXI, enmarcado en la Conferencia de Naciones Unidas sobre Medio Ambiente y Desarrollo que se celebró en la Cumbre de la Tierra de Río de Janeiro, en junio de 1992. En esta cumbre se asume el término Desarrollo Sostenible que fue definido previamente

* Javier Martínez Vega y María Asunción Martín Lou. Dpto. de Geografía. Instituto de Economía y Geografía (CSIC).

Estudios Geográficos, LXIII, 248/249, 2002 
en el Informe de la Comisión Mundial sobre Medio Ambiente y Desarrollo de 1987, más conocido como Informe Brundtland.

En el capítulo 28 del programa de Río, conocido como Programa 21, se hace un llamamiento a las autoridades locales para que elaboren Agendas 21 Locales, buscando la participación de los ciudadanos y consenso para la implantación de estrategias de desarrollo sostenible al nivel local (Naciones Unidas, 1992). Las Agendas 21 son Planes de Acción Local promovidos por las entidades locales, comarcales o regionales y se fundamentan en el principio de responsabilidad compartida y colaboración.

En mayo de 1994 se firma la Carta de ciudades europeas hacia la sostenibilidad, más conocida como Carta de Aalborg. En ella, las ciudades y municipios europeos firmantes se comprometen a integrar los principios de Sostenibilidad y Justicia Social en las políticas económica, social y ambiental a todos los niveles y, en especial, en el ámbito local mediante procesos de planificación y gestión diseñados por los propios municipios.

En 1996, la Carta de Lisboa y, más recientemente, en febrero de 2000 la Declaración de Hannover evalúan y revisan los principios de Sostenibilidad y las experiencias desarrolladas hasta entonces. Las entidades municipales continúan realizando un llamamiento a la comunidad internacional, a las instituciones europeas, a los gobiernos y otras instituciones para la promoción y su implicación en el proceso de la Agenda 21.

El VI Programa de Acción en materia de Medio Ambiente de la UE (2001-2010) propone diseñar un Programa Marco, jurídicamente vinculante, basado en los principios de «cautela», «acción preventiva» y «quien contamina paga». Este programa se debe completar con Planes de Acción nacionales en materia de Medio Ambiente en cada estado miembro.

De forma concurrente, puede decirse que la Ordenación del Territorio es la «expresión espacial de la política económica, social, cultural y ecológica de toda la sociedad, cuyos objetivos fundamentales son el desarrollo socioeconómico y equilibrado de las regiones, la mejora de la calidad de vida, la gestión responsable de los recursos naturales, la protección del medio ambiente y, por último, la utilización racional del territorio» (Carta Europea de Ordenación del Territorio, 1983).

Por ese motivo, también la Ordenación del Territorio persigue la definición de un modelo futuro de territorio en el que se implanten

Estudios Geográficos, LXIII, 248/249, 2002

$$
-712-
$$


las actividades y aprovechamientos propiciados por las políticas sectoriales acordadas por la sociedad, con el objetivo de alcanzar un Desarrollo sostenible en ese territorio (Gómez Orea, 2001). Esta idea de futuro justifica el carácter orientativo que caracteriza a la ordenación territorial.

Como ya es sabido, en España las competencias en materia de ordenación del territorio están transferidas a las Comunidades Autónomas en virtud del artículo 148 de la Constitución Española. En consecuencia, desde 1983 se han venido promulgando leyes de ordenación del territorio de ámbito autonómico, acordes con las singularidades de cada territorio. En Castilla-La Mancha, la Ley 2/1998 de 4 de junio de Ordenación del Territorio y Urbanismo regula estas materias con criterios (art. 4) de desarrollo racional y equilibrado de las actividades en el territorio $(a)$, armonización de los requerimientos del desarrollo económico y social con la preservación y mejora del medio ambiente urbano y natural, asegurando a todos una digna calidad de vida $(b)$ y de promoción de la cohesión e integración sociales, así como de la solidaridad regional, intermunicipal y municipal (c). Por otra parte, los artículos 5 y 6 indican los fines de toda actuación pública territorial y de la actividad pública urbanística, respectivamente, de acuerdo a los principios de sostenibilidad, solidaridad territorial y cohesión social.

Esta convergencia entre Ordenación del Territorio y el Desarrollo Sostenible queda claramente manifiesta en el documento de consulta para la Estrategia Española de Desarrollo Sostenible (EEDS) que el Ministerio de Medio Ambiente ha elaborado de forma provisional (diciembre de 2001) y sobre el que está pidiendo informes a distintos interlocutores sociales y económicos (CES, 2002). Entre los instrumentos de la EEDS se señala la necesidad de la integración del desarrollo sostenible en políticas como la de Ordenación del Territorio, especialmente en España con fuerte polarización y desequilibrios territoriales. Para alcanzar el objetivo de utilizar racionalmente el suelo se establecen algunas medidas como el estímulo de una política de desarrollo regional que favorezca la ocupación equilibrada del territorio, la incorporación de evaluaciones ambientales de las políticas sectoriales e incentivar el desarrollo en las regiones más desfavorecidas. Conviene indicar que el Consejo Europeo de Gotemburgo, junio de 2001, adoptó la Estrategia de Desarrollo Sostenible de la Unión Europea que sirve de marco legal a la EEDS.

Estudios Geográficos, LXIII, 248/249, 2002 


\section{Antecedentes en España}

En España existen acciones encaminadas a la puesta en marcha de la Agenda 21, repartidas por todo el territorio nacional. En este apartado se reseñarán tan sólo algunas de las más relevantes.

La Carta de Valencia - también llamada la Carta de las nacionalidades y de las regiones europeas para el medio ambiente, elaborada en la II Conferencia de Ministros y Responsables Políticos de las Regiones de la Unión Europea en materia de Medio Ambiente, celebrada en Valencia, el 21 de noviembre de 1995- es el germen de la Declaración de Xátiva, de mayo de 2000. En ella, se crea la Red de Municipios Valencianos hacia la Sostenibilidad (DVA, 2002). Esta declaración reflexiona sobre los siguientes aspectos:

- Una profundización en el planteamiento de los temas medioambientales de carácter netamente municipalista, en la que la cooperación y la asistencia técnica entre la Diputación y los Ayuntamientos de la Provincia, en coordinación con la Generalitat Valenciana, impulse un Desarrollo Sostenible para nuestros municipios de acuerdo con los objetivos programados de la Agenda 21.

- Establecer convenios entre los Municipios de la Provincia y la Diputación a fin de propiciar la realización de auditorias medioambientales que sirvan de base para la redacción de los planes de acción local.

- Incentivar la creación de la Red de Municipios Valencianos hacia la Sostenibilidad, en la cual además de la Diputación se integren los Ayuntamientos, y otras Entidades públicas o privadas que voluntariamente lo decidan y cuyo objetivo central será el de contribuir a la aplicación de políticas municipales sostenibles.

En la actualidad la relación de Ayuntamientos adheridos es de 113, y en trámites de adhesión a la red de municipios valencianos 58 municipios.

Uno de los instrumentos fundamentales para la elaboración de las Agendas 21 son las llamadas ecoauditorías municipales. Éstas pretenden dotar a los municipios de una herramienta eficaz, concreta y operativa, que sirva para favorecer el desarrollo de las capacidades económicas locales, basándose en la utilización sostenible de los recursos (DVA, 2002).

Estudios Geográficos, LXIII, 248/249, 2002

$$
-714-
$$


La encuesta municipal de Medio Ambiente (EMMA) es un instrumento de gran ayuda en la etapa de análisis y diagnóstico territorial.

En Cataluña, en octubre de 1997, el Parlamento instó al Gobierno de la Generalidad de Cataluña a potenciar los trabajos para elaborar la Agenda 21 de Cataluña, estableciendo unos mecanismos que favorezcan la participación de los agentes sociales en el proceso de reflexión, incluyendo un equipo de expertos (GENCAT, 2002).

Un panel de más de 100 expertos, representativos de los diversos sectores de la sociedad catalana, constituye el Forum Consultivo para la Agenda 21 de Cataluña. Para los trabajos, se parte de un Documento cero que es un primer guión de lo que deberá ser la Agenda 21 de Cataluña. Durante meses, el Departamento de Medio Ambiente, junto con otros departamentos de la Generalitat y con el Forum consultivo, desarrollaron este Documento cero hasta llegar al texto final.

Fruto de esta iniciativa surgen algunos ejemplos relevantes de la implantanción de la Agenda 21 en municipios catalanes:

- Diputación de Barcelona: La creación de la Red Catalana de Ciudades y Pueblos sostenibles, impulsada por la Diputación de Barcelona y después por la misma Generalitat, puso a la provincia de Barcelona como pionera en Europa. Con un crecimiento espectacular de la cantidad de ciudades que la forman, 150 pueblos y ciudades de todos los tamaños y colores políticos y de características sociales muy diferentes, esta red y sus grupos de trabajo están elaborando las auditorías ambientales para confeccionar los Planes de acción de las Agendas 21, una colección de una cincuentena de indicadores de estado, presión y respuesta y unos trabajos sectoriales sobre flujos de energía, del ciclo de agua, de los transportes y de los residuos, que son considerados ejemplares por la comunidad científica y por las instituciones europeas responsables del medio ambiente.

- La Agenda 21 del Bages es un plan estratégico que define las acciones de mejora ambiental a realizar para situar la evolución futura de la comarca en la línea del desarrollo sostenible. La configuración de esta agenda puede considerarse como un esfuerzo novedoso y piloto dentro de la aplicación de estos programas en Cataluña. Desde el año 1994, se llevan a cabo diversas aplicaciones a escala municipal que tienen como principal limitación la falta de articulación en- 
tre las escalas territoriales de análisis y propuesta; esta limitación se manifiesta en la falta de cohesión entre la escala comarcal y la escala local.

Para superar estas limitaciones, el Departamento de Medio Ambiente de la Generalidad de Cataluña y el Consejo Comarcal del Bages firmaron en febrero de 1998 un acuerdo para redactar una propuesta inicial de Agenda 21 del Bages. En el mes de septiembre de 1998, el Pleno del Consejo Comarcal del Bages aprobó la realización de la agenda siguiendo el método y los contenidos que define el documento inicial, que incluye una propuesta de objetivos, un método y un esquema de participación ciudadana.

La propuesta se está terminando de perfilar y, en el mes de enero de 1999, se hizo una amplia difusión de la misma para que todas las administraciones, entidades, asociaciones y personas de la comarca pudieran aportar sus opiniones y propuestas para mejorar su contenido y su eficacia.

De esta forma, y para afrontar el nuevo siglo, la comarca gozará de una ordenación temporal y espacial de la intervención integrada y coordinada de las administraciones para mejorar el medio ambiente.

El método de trabajo utilizado para la formulación de la propuesta parte de un marco general interpretativo de las relaciones entre sociedad y medio ambiente de la comarca, que se fundamenta en tres pilares esenciales: en primer lugar, la recogida y sistematización cartográfica, evolutiva, conceptual y estadística de la información necesaria; en segundo lugar, el diagnóstico y prioridad de las problemáticas y, en tercer lugar, la definición de objetivos para cada problemática prioritaria así como la concreción de las opciones y estrategias básicas para alcanzar los objetivos fijados.

La efectividad y la verdadera fuerza de cualquier agenda reside en la asunción, por parte de la mayoría de los agentes sociales de la comarca, de sus objetivos, estrategias y propuestas de actuación; por ello uno de los ejes básicos para asegurar el cumplimiento de los compromisos de la Agenda es el plan de comunicación. Éste pretende asegurar, desde un principio, el conocimiento de las características ambientales esenciales de la comarca, la difusión de las mismas y la participación de los ciudadanos en la elaboración de la Agenda de actuaciones. Este plan se fundamenta y se activa sobre tres pilares básicos: el contacto permanente con los medios de comunicación, la realización de jornadas de debate es- 
pecíficas y el funcionamiento del web de la Agenda 21 del Bages. Estos fundamentos estarán activos durante todo el proceso de elaboración y aprobación de la Agenda y facilitarán la participación ciudadana.

Otra iniciativa institucional es la llevada a cabo por el departamento de Medio Ambiente del Gobierno de Navarra que trata de impulsar la elaboración de las Agendas 21 Locales en todos aquellos municipios mayores de 4.000 habitantes. Para alcanzar este fin, el gobierno autonómico aporta recursos financieros propios. En los últimos años se estaban elaborando las Agendas 21 Locales de 16 municipios -Pamplona, Cintruénigo, Tudela, Tafalla, Estella, entre otros-y de la Mancomunidad de Aragón y de los Valles Subcantábricos (Gobierno de Navarra, 2002). El Departamento de Medio Ambiente, Ordenación del Territorio y Vivienda ha propiciado la formación de una Red de Ciudades y Pueblos Navarros hacia la Sostenibilidad. La metodología se apoya en la elaboración de auditorías ambientales como herramienta fundamental en la etapa de análisis y diagnóstico de los municipios y consorcios, tales como comarcas naturales y agrupaciones dispersas.

La Fundación Desarrollo y Naturaleza (Deyna, 1997), también ha propiciado la implantación de un buen número de Agendas 21 Locales. Con una metodología muy sencilla, indica los pasos a seguir por las corporaciones locales y los representantes de los agentes sociales y económicos de los municipios para realizar una Agenda 21 Local. Este método no considera necesaria la elaboración de auditorías medioambientales sino que persigue la elaboración de una lista priorizada de problemas y líneas de acción, consensuada por la población local y asumida por la corporación como plan de acción local. Se ha elaborado en los 183 municipios de la provincia de Soria y se ha presentado en la Exposición de Hannover 2000 como un proyecto de demostración de interés mundial. Obra en poder de UNESCO, del PNUMA. La Agencia Europea de Medio Ambiente (EEB) de la UE la expuso en el Congreso del ICLEI de 1997 en Estocolmo.

Haciendo un paréntesis, conviene decir que el Consejo Internacional de Iniciativas Locales para el Medio Ambiente (ICLEI) elaboró en 1998 una guía europea para la planificación de las agendas locales 21 que es una referencia obligada (Hewwitt, 1998).

La Junta de Andalucía (2000) ha elaborado un documento de reflexión que sienta las Bases para la Agenda 21 en Andalucía (Junta de Andalucía, 2000). En esa misma Comunidad Autónoma se está elaborando la Agenda 21 del litoral de la Janda (Barragán, 2000). Se trata de una agen-

Estudios Geográficos, LXIII, 248/249, 2002 
da sobre la Mancomunidad de municipios de la Janda - Barbate, Conil y Vejer-, dirigida por un grupo de investigación de la Universidad de Cádiz. Se trata de un documento muy riguroso desde el punto de vista científico. Actualmente, este mismo grupo está elaborando la Agenda 21 de la Costa Noroeste (UCA, 2002) en la Mancomunidad de municipios gaditanos del Bajo Guadalquivir - Sanlúcar de Barrameda, Rota, Chipiona y Trebujena-. En ambos casos, se trata de agrupaciones de municipios en zonas litorales por lo que el estudio del medio ambiente terrestre, marino y de la zona de contacto otorga complejidad en la etapa de análisis y diagnóstico territorial.

El Cabildo de Lanzarote ha publicado la estrategia de Lanzarote en la Biosfera (Prats y Miguélez, 1998) como documento para el debate. En él se reflexiona sobre las estrategias deseables para alcanzar el objetivo del desarrollo sostenible de la isla que está catalogada toda ella como Reserva de la Biosfera. Una de las iniciativas es concederse una moratoria hasta el año 2007 para evaluar los costes ambientales del modelo de desarrollo turístico que se seguía hasta ahora. Este documento se considera como la base de una Agenda 21.

Finalmente, cabe mencionar el documento que ha publicado el Centro de Estudios Ambientales (1998) del Ayuntamiento de Vitoria en el que se define la Agenda 21 de la ciudad siguiendo el modelo del ICLEI, el Instituto Internacional de Iniciativas Locales para el Medio Ambiente.

Otro ejemplo de Agenda 21 en municipios urbanos es el de Valladolid. La auditoría ambiental la han elaborado los técnicos de los distintos departamentos del ayuntamiento. La agenda se estructura en torno a 18 programas sectoriales bajo los cuales se proponen una serie de acciones específicas y de compromisos a alcanzar a corto, medio y largo plazo. Para su ejecución no se prevén aportaciones financieras adicionales sino que se piensan acometer las acciones propuestas aprovechando los recursos financieros ordinarios del propio ayuntamiento.

En la Comunidad de Madrid, el municipio de Madrid se encuentra en el proceso de elaboración de su Agenda 21. En el mes de junio de 2002 se celebraron unas jornadas técnicas que perseguían el intercambio de experiencias en ciudades europeas de tamaño medio y grande. En otros municipios metropolitanos la situación es desigual. Galapagar se encuentra en la fase de ejecución de la auditoría ambiental mientras que otros, como Torrelodones, han firmado la Carta de Aalborg pero aún no han iniciado los trabajos de las ecoauditorías.

Estudios Geográficos, LXIII, 248/249, 2002

$$
-718-
$$


En Castilla-La Mancha, la Federación de Municipios y Provincias de esa Comunidad Autónoma ha solicitado la colaboración del Gobierno regional para confeccionar una auditoría de la situación ambiental de los pueblos castellano-manchegos e impulsar las sostenibilidad de sus ciudades, de acuerdo a los objetivos de la Carta de Aalborg (Geoscopio, 2002). Al Pacto de Ciudades Habitables se han adherido 112 ciudades y municipios, entre los cuales figuran las capitales de Albacete, Cuenca y Guadalajara.

En resumen, existen distintas experiencias previas que sirven de referencia a nuevos proyectos de implantación de agendas 21 en España.

\section{Objetivos}

El objetivo principal de este proyecto es la elaboración de una propuesta de Agenda 21 Comarcal cuyo ámbito territorial es la Mancha Alta Conquense que más adelante se definirá. Esta Agenda 21 tiene como finalidad los siguientes objetivos:

1. Elaboración de una Auditoría Medioambiental de la comarca, entendida como un instrumento objetivo de análisis y diagnóstico territorial y como herramienta de ayuda para establecer las estrategias de acción local derivadas. Para alcanzar este objetivo es necesario, previamente:

a. Efectuar un Análisis territorial de la comarca con objeto de inventariar los recursos naturales estratégicos y evaluar su estado de conservación, de conocer las principales características de la población y de sus actividades socioeconómicas y de estudiar los principales vectores ambientales.

$b$. Realizar un Diagnóstico territorial con el fin de identificar las principales debilidades, amenazas, fortalezas y oportunidades del territorio.

2. Determinación de objetivos de sostenibilidad factibles y concretos con el objetivo de resolver o atenuar los problemas ambientales y socioeconómicos del territorio en función de las fortalezas y oportunidades del mismo. Se pretende disminuir las disfuncionalidades del sistema territorial. Una vez determinados los objetivos, se procederá a la definición de líneas estratégicas de actuación priorita-

Estudios Geográficos, LXIII, 248/249, 2002

$$
-719-
$$


ria y a sugerir Planes de Acción Local específicos según las zonas ambientalmente homogéneas que puedan ser delimitadas en la propuesta de Agenda 21 Comarcal.

3. Elaboración de un Sistema de Información Geográfica Comarcal concebido como un producto añadido de la propuesta de Agenda 21 Comarcal y como una herramienta de ayuda y orientación en el complejo proceso de toma de decisiones por parte de las autoridades locales, del grupo de acción local o de los distintos grupos de agentes sociales y económicos de la comarca.

\section{Ámbito territorial}

El área de estudio es la comarca de la Mancha Alta Conquense que ocupa una superficie de $1.824 \mathrm{Km}^{2}$, repartida sobre el territorio de $29 \mathrm{mu}-$ nicipios del W de la provincia de Cuenca (Figura 1).

En medio de las vastas parameras cultivadas se encuentran complejos lagunares como las lagunas de El Hito o Navahonda que poseen elevado

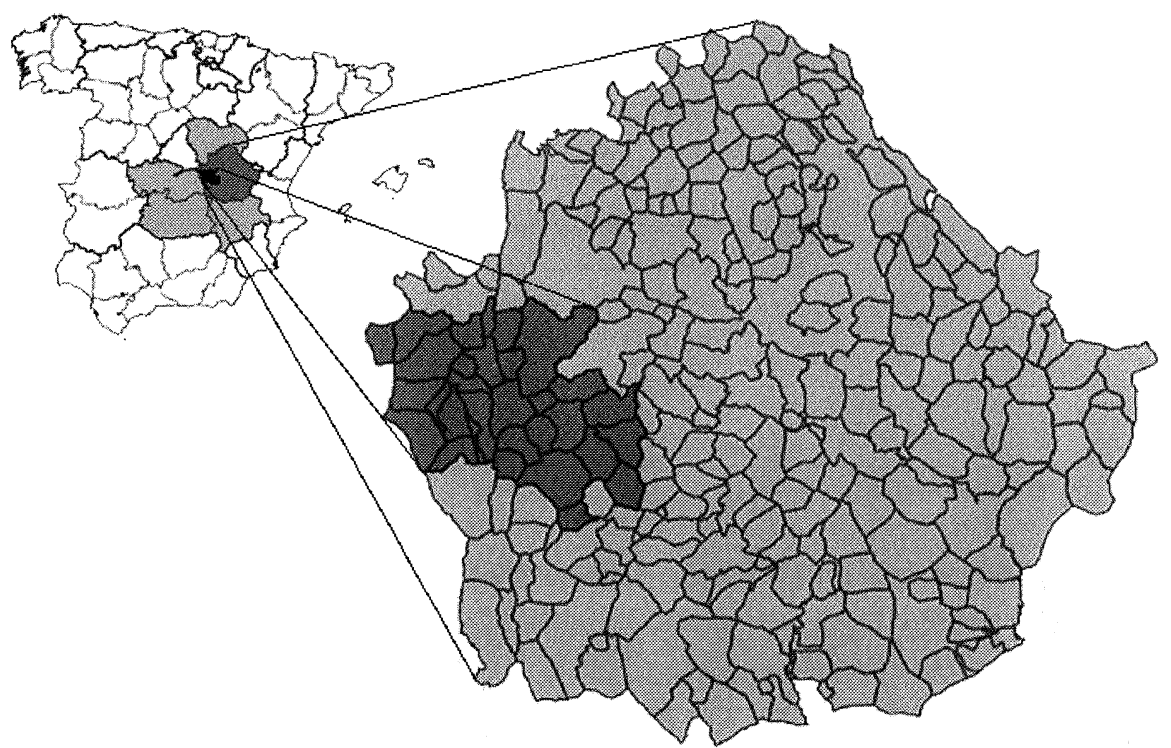

FIg. 1.-Localización del área de estudio: La Mancha Alta Conquense.

Estudios Geográficos, LXIII, 248/249, 2002 
interés ambiental ya que son hábitat de especies florísticas y faunísticas de interés internacional. Las estepas cerealistas también son hábitats singulares para las aves esteparias por lo que formarán parte de la red ecológica de la UE, Natura 2000. Esta diversidad biológica es demostrativa de la viabilidad coherente del desarrollo de las actividades agrarias con la conservación de la naturaleza.

La población que habita esta comarca, según los datos del Padrón de 1 de enero de 2000, es de 27.229 habitantes. Este hecho supone una débil densidad demográfica en la comarca $\left(14,9 \mathrm{hab} . / \mathrm{km}^{2}\right)$, inferior a la media regional -Castilla-La Mancha poseía una densidad de $21,5 \mathrm{hab} . / \mathrm{km}^{2} \mathrm{en}$ 1997- que es una de las más bajas de todas las regiones de la UE, exceptuando las regiones escandinavas. Por otra parte, los efectivos demográficos se encuentran desigualmente repartidos sobre el territorio comarcal. Tarancón concentra el $42 \%$ de la población y Horcajo de Santiago otro $13 \%$.

$\mathrm{Al}$ estar enclavada la comarca estudiada en la Comunidad Autónoma de Castilla-La Mancha y poseer esta última un PIB inferior al $75 \%$ respecto a la media comunitaria (Castilla-La Mancha poseía en 1997 un PIB de 54,1 respecto a la media de la $\mathrm{UE}=100$ ), todo el territorio comarcal es una zona considerada objetivo 1 .

Es evidente que, a pesar de estas debilidades estructurales, el territorio también posee potencialidades y oportunidades que deben ser aprovechadas con objeto de potenciar el desarrollo socioeconómico y la cohesión social, objetivo del Programa 21 y de todas las estrategias y políticas de ordenación territorial y desarrollo sostenible.

\section{Enfoque y Metodología}

El enfoque general a seguir es ascendente — bottom-up-, basado en un proceso participativo de la población local, tal como recomienda el Programa 21 de Naciones Unidas y como indica la Carta de Aalborg:

"Nosotras, las ciudades, nos comprometemos a seguir el mandato de la Agenda 21, documento clave aprobado en la Cumbre de la Tierra en Río de Janeiro, de trabajar con todos los sectores de nuestras comunidades - ciudadanía, empresas, grupos de interés-en el desarrollo de las Agendas 21 locales. Estamos de acuerdo con el lla-

\section{$-721-$}

Estudios Geográficos, LXIII, 248/249, 2002 
mamiento del Quinto Programa de Acción en materia de medio ambiente de la Unión Europea: "Hacia un desarrollo sostenible» y a compartir, por tanto, la responsabilidad de la aplicación del programa en todos los sectores de la comunidad. En consecuencia, basaremos nuestros trabajos en la cooperación entre todas las partes implicadas. Garantizaremos el acceso a la información de toda la ciudadanía y de los grupos que estén interesados y velaremos para que puedan participar en los procesos locales de toma de decisiones. Buscaremos oportunidades de educación y formación en materia de sostenibilidad, no sólo para el público en general sino también para los representantes elegidos y el personal de las administraciones locales».

Punto 1.13 de la Declaración de Consenso (Parte I) de la Carta de Aalborg (1994)

Se prevé que la participación de la población local se materialice a través de múltiples vías: mediante la incorporación de expertos locales, técnicos municipales, técnicos de desarrollo rural y otros conocedores de la realidad territorial en el equipo técnico que aborda la auditoría medioambiental; colaborando, como encuestadores y encuestados, en los sondeos de opinión que se hagan sobre los problemas ambientales, sociales y económicos y sobre las vías de solución que pueden identificarse en relación con la determinación de objetivos previa al Plan de Acción Local; participando en los grupos de trabajo específicos como expertos o técnicos; siendo informados sobre los impactos - ambientales, sociales y económicos- de las actuaciones señaladas como preferentes dentro del mencionado Plan.

Así pues, se asegura la incorporación de la opinión de la población local en la elaboración del diagnóstico comarcal y en la definición de las líneas estratégicas de actuación, permitiendo tener una visión nueva, práctica y enriquecedora de la realidad de la comarca. Esta participación de la población local en la definición de un modelo territorial de futuro requiere una reflexión, sensibilización, compromiso y responsabilidad de acciones futuras.

Aunque se está recurriendo a una metodología ad hoc, este trabajo se inspira en procedimientos empleados en trabajos precedentes, entre los que queremos destacar los utilizados por la Diputación de Barcelona, por el Gobierno de Navarra (2002) y por la Universidad de Cádiz (Barragán,

Estudios Geográficos, LXIII, 248/249, 2002

$$
-722-
$$


2000; UCA, 2002). Así pues, para el desarrollo de este proyecto se prevén tres fases de trabajo: I. Fase preparativa, II. Fase de Auditoría Medioambiental y III. Fase de diseño del Plan de Acción Local.

En la Fase I, se han seleccionado los municipios que están siendo objeto de la auditoría ambiental y del Plan de Acción Local. En esta fase se han firmado los acuerdos y convenios necesarios entre el Grupo de Acción Local y el Equipo Técnico que desarrolla el proyecto, y se están negociando otros acuerdos entre el primero y otras instituciones locales, provinciales y autonómicas que están interesadas en el proyecto. La composición del Equipo Técnico es multidisciplinar y se estudia la conveniencia de incluir, como ya se ha dicho, expertos y técnicos locales de las instituciones y entidades involucradas: ayuntamientos, mancomunidades, Diputación de Cuenca, Junta de Comunidades de Castilla-La Mancha, etc. Finalmente, se ha planificado el proceso de trabajo de acuerdo a un cronograma de tareas, se han asignado responsabilidades y se ha fijado un calendario de reuniones que se celebran regularmente a distintos niveles: entre los participantes del equipo técnico, entre los miembros de la comisión ejecutiva, entre los componentes del foro de debate y se prevén reuniones de los grupos de trabajo sectoriales, una vez que se constituyan.

En la Fase II, se está realizando una Auditoría Medioambiental. Se está procediendo a la recopilación de información sobre las variables ambientales, sociales y económicas que se consideran estratégicas para conocer el funcionamiento y organización del sistema territorial (Figura 2).

Se hará un tratamiento de dichas variables mediante diversos métodos de análisis espacial, con un enfoque cuantitativo, y de investigación social, con un enfoque cualitativo, con objeto de facilitar el establecimiento de un diagnóstico territorial. Se elaborará una matriz DAFO para identificar las debilidades, amenazas, fortalezas y oportunidades del territorio e identificar las estrategias de acción local que sean más convenientes en cada caso.

Finalmente, se procederá a la delimitación de áreas ambientales homogéneas con el fin de definir, en la medida de lo posible, planes de acción local específicos en cada área.

En la Fase III se determinarán los objetivos de acuerdo a un orden de prioridad establecido y de las políticas y criterios de gestión de las distintas administraciones públicas que tengan incidencia sobre el territorio.

Estudios Geográficos, LXIII, 248/249, 2002 


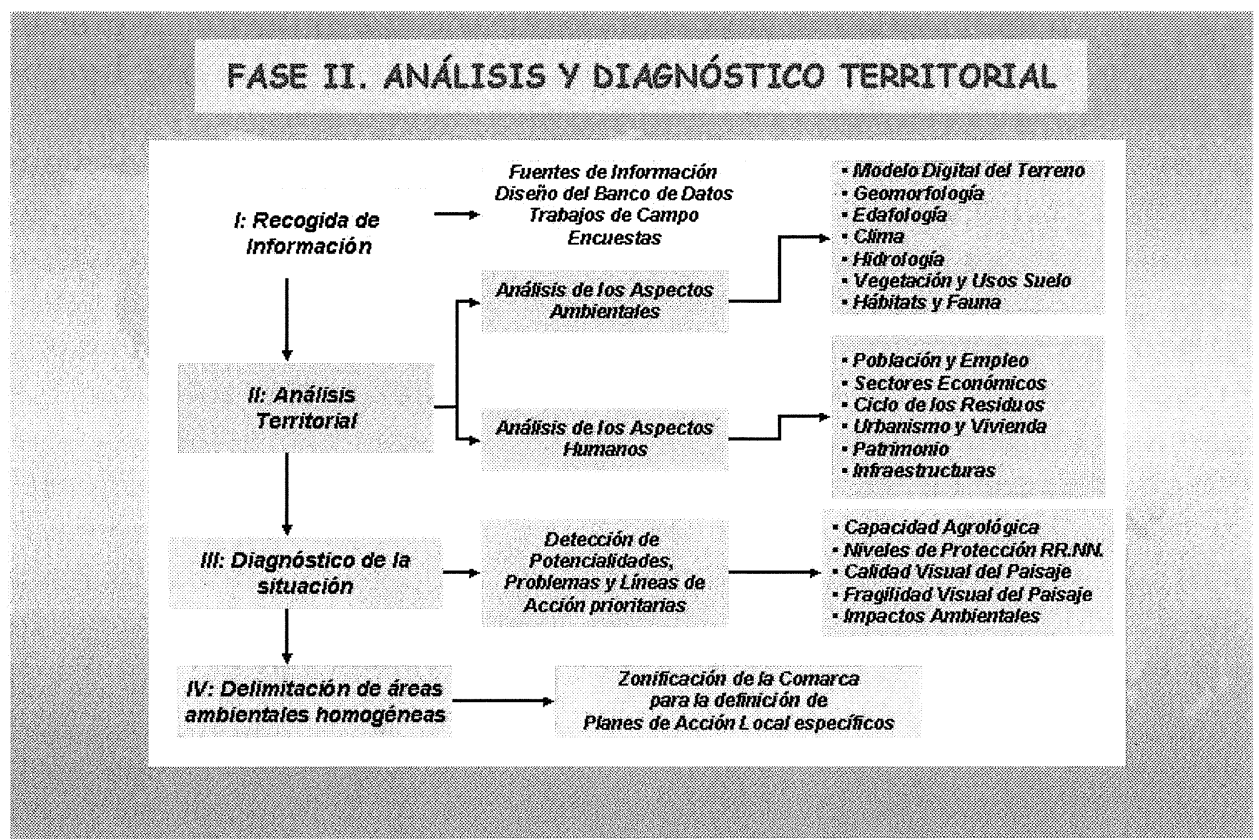

FIG. 2.-Esquema de la fase de la auditoría medioambiental.

Con la ayuda del Sistema de Información Geográfica de la comarca y, tras la consulta de las prioridades de la población local, se propondrán una serie de propuestas en cada una de las zonas en que se haya dividido la comarca. Se espera que estas propuestas fundamentarán los Planes de Acción Local.

\section{Estructura institucional y operativa}

Para que el proceso de elaboración de la Agenda 21 sea eficiente y siguiendo experiencias previas (Barragán, 2000), se ha propuesto que particulares y organismos participantes se organicen de acuerdo a la estructura institucional y operativa que se esquematiza en la Figura 3.

Una Comisión Ejecutiva, formada por representantes del Grupo de Acción Local y del Equipo Técnico, que se encarga de coordinar los trabajos de la Agenda 21 y de efectuar el seguimiento de cada fase del proyecto.

Estudios Geográficos, LXIII, 248/249, 2002 


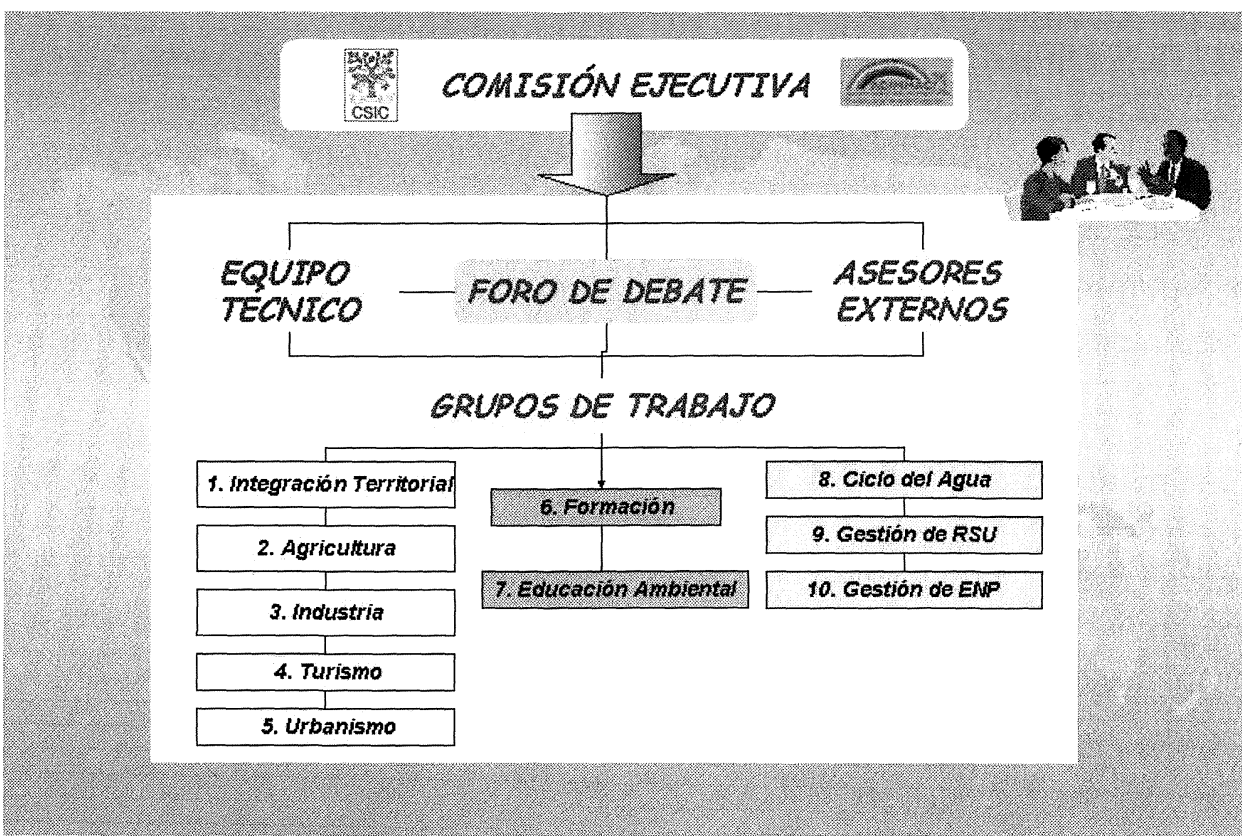

FIG. 3.-Estructura institucional y operativa de la Agenda 21.

Un Equipo Técnico que está formado por científicos y profesores de distintas disciplinas. Como ya se ha indicado, sería conveniente que se integraran en él todos aquellos técnicos de los municipios, de las Mancomunidades existentes en La Mancha Alta Conquense, de la Diputación de Cuenca, de la Junta de Comunidades de Castilla-La Mancha y del programa PRODER que estén interesados en colaborar en el desarrollo de la Agenda 21. Su función principal es elaborar la auditoría medioambiental y proporcionar información y orientación técnica al Foro de Debate y a los Grupos de Trabajo durante el proceso para facilitar la toma de decisiones.

Un Foro de Debate que está compuesto por representantes de todas las administraciones públicas, otras instituciones públicas y privadas, de las asociaciones y entidades vinculadas con el medio natural y con el desarrollo social y económico de la comarca. Han sido designados de forma global, procurando que la representación sea lo más amplia y plural posible. Se le encomienda la responsabilidad de participar en todo el proceso de elaboración de la Agenda 21 y de señalar los principales temas clave o programas que van a constituir los grupos de trabajo sectoriales o temá-

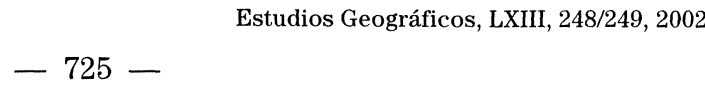


ticos. Además, deben aportar ideas y opiniones sobre el diagnóstico y sobre los objetivos y acciones estratégicas que se deriven de cada programa. Su misión principal es aprobar el documento final de la Agenda 21.

Unos Grupos de Trabajo específicos sobre los temas clave que designe el foro de debate, compuestos, de forma orientativa, por una decena o docena de especialistas y técnicos expertos en cada uno de los temas. Ellos deben proponer, lo más detalladamente posible, los objetivos y acciones estratégicas que se consideren prioritarios en cada programa temático.

Asesores Externos - técnicos, investigadores, gestores y especialistas en distintas materias, especialmente en aquellas relacionadas con los programas designados-serán consultados sobre cuestiones puntuales que se requieran para afinar el diagnóstico y analizar los resultados de los distintos grupos de trabajo y expresar su opinión sobre los mismos.

\section{Estado actual: resultados provisionales}

La Agenda 21 de la comarca Mancha Alta Conquense se encuentra en la segunda fase de trabajo. Equipo técnico y población local participan en la elaboración de la auditoría medioambiental como herramienta eficaz para realizar un análisis territorial riguroso y para proporcionar un diagnóstico operativo sobre la comarca.

Dentro de esta etapa, se está recopilando la información a partir de fuentes primarias y secundarias y se está diseñando un Sistema de Información Geográfica Comarcal. El Centro Geográfico del Ejército ha proporcionado la base cartográfica numérica (BCN) de la comarca, a escala $1 / 50.000$, en formato *dgn. Planimetría, altimetría y toponimia han sido empleadas para la formación del mapa topográfico de la comarca, a partir del cual podrá extraerse otra información geográfica de interés mediante análisis espacial. Asimismo, este mapa de base servirá de soporte de la información temática para la formación de mapas analíticos y sintéticos.

En cuanto al análisis de los aspectos ambientales, se ha elaborado el Modelo Digital de Elevaciones (MDE) a partir de las curvas de nivel y de los puntos acotados de la BCN50. Mediante análisis espacial se ha derivado un mapa hipsométrico y se está trabajando para obtener mapas de pendientes y de orientaciones.

Estudios Geográficos, LXIII, 248/249, 2002 
Mediante trabajos de campo, fotointerpretación de estereogramas y la reinterpretación de mapas geológicos ya existentes se están formando el mapa geológico, de litología y de suelos de la comarca.

El Instituto Meteorológico Nacional ya nos ha proporcionado la base de datos climáticos de las estaciones meteorológicas de la comarca y de su entorno. Estos datos, correlacionados con el MDE y con datos de latitud y longitud, permitirán modelar espacialmente variables biofísicas relevantes para ser incluidas en modelos de capacidad de uso, por ejemplo.

Se dispone ya del mapa de cultivos y aprovechamientos de la comarca en formato digital, aunque se prevé su actualización mediante la interpretación de una escena Landsat ETM+ de este verano. Su comparación permitirá conocer la estructura de los cambios producidos en los usos del suelo y formular patrones de cambio, tendencias y aplicar índices de ecología del paisaje. Por otra parte, la ocupación biofísica del territorio es una variable estratégica para derivar estudios de la calidad visual y fragilidad del paisaje comarcal y para asignar niveles de protección a los hábitats y a las especies de fauna.

La Dirección General de Conservación de la Naturaleza nos ha proporcionado la cobertura y las fichas de los Lugares de Interés Comunitario, presentes en la comarca, propuestos por la Junta de Comunidades de Castilla-La Mancha para formar parte de la Red Natura 2000.

Entre los datos socioeconómicos más relevantes se dispone de una base de datos del padrón municipal de enero de 2000 que pone a disposición la Junta de Comunidades de Castilla-La Mancha a todos los visitantes de su web (J.CC. Castilla-La Mancha, 2002).

Por último, la oficina técnica del programa PRODER nos ha facilitado otros datos socioeconómicos de gran interés. Entre otros, se dispone de un directorio de industrias, comercios, servicios, equipamientos e infraestructuras radicados en cada municipio.

De forma paralela, el Equipo Técnico ha elaborado una encuesta durante el mes de octubre de 2002 con objeto de conocer las opiniones, las actitudes y la visión de futuro de los líderes locales de la comarca que forman parte del Foro y, más adelante, de los Grupos de Trabajo específicos. En definitiva, se dirige a identificar la percepción de problemas y oportunidades de la comarca. Este es uno de los planos en los que se evidencia la importancia de la participación ciudadana. La búsqueda del consenso en el diseño de una estrategia de futuro es esencial.

Estudios Geográficos, LXIII, 248/249, 2002 
Esta encuesta consta de 82 preguntas y se estructura en tres partes. Las dos primeras se refieren al Medio Natural y a la Dinámica socioeconómica del conjunto de la comarca. Se pretende identificar los recursos naturales que pueden dinamizar el desarrollo sostenible y los impactos generados sobre el medio ambiente, conocer el funcionamiento de las actividades sectoriales y la capacidad de la población local para emprender nuevas actividades, implantar nuevos cultivos. Además, se procura conocer cuáles son los equipamientos que la población local considera más relevantes para un desarrollo sostenible de la comarca y el grado de sentimiento de pertenencia a ésta.

La tercera parte se dirige a conocer la problemática ambiental y socioeconómica así como las potencialidades del municipio en el que reside cada encuestado. Se intenta identificar los principales problemas urbanísticos, la disponibilidad de instrumentos de planeamiento urbanístico y las preferencias de los vecinos ante el futuro urbanístico, su grado de satisfacción en relación a los servicios, equipamientos e infraestructuras, el funcionamiento de los ciclos del agua, de los residuos sólidos urbanos y conocer los puntos de contaminación.

Se han realizado un total de 80 encuestas a los líderes de la comarca que forman parte del Foro de Debate, considerando que esta muestra es significativa de la población comarcal, no tanto en términos estadísticos sino porque aquéllos son los representantes cualificados de los distintos sectores de la sociedad local. Como ya se ha dicho, se ha procurado que los miembros del Foro sea una muestra lo más representativa y plural posible. Entre ellos, figuran los representantes políticos de todas las administraciones con implantación territorial, desde las corporaciones locales hasta la Administración General del Estado, pasando por las entidades públicas sectoriales. Junto a ellos, se encuentran representantes del Grupo de Acción Local, de la oficina técnica del PRODER, de ONGs, de asociaciones de empresarios, organizaciones agrarias, cooperativas, oficinas comarcales agrarias, cajas de ahorro con implantación en la comarca y un largo etcétera.

En el momento actual, el Equipo Técnico se encuentra tabulando los resultados de las encuestas con objeto de extraer las conclusiones pertinentes y disponer de una base de conocimientos suficiente para elaborar un pre-diagnóstico operativo de la comarca.

En el mes de octubre de 2002 se constituyó formalmente el Foro de Debate y se eligieron al presidente, vicepresidente y secretario del mismo.

Estudios Geográficos, LXIII, 248/249, 2002

$$
-728-
$$


Próximamente los componentes del Foro decidirán los grupos de trabajo que se considerarán prioritarios, a la vista de los resultados de la encuesta y del pre-diagnóstico operativo.

\section{Conclusiones}

Se evidencia, en los últimos años, una convergencia cada vez mayor entre las estrategias y políticas sobre desarrollo sostenible, medio ambiente, ordenación del territorio y desarrollo rural. Esta aproximación se produce tanto a nivel global como continental, nacional y regional, al menos en los ámbitos territoriales de la Unión Europea, de España y de nuestras Comunidades Autónomas.

Como se ha visto, las iniciativas para elaborar Agendas 21 son numerosas en España. Desde el punto de vista espacial, existen ejemplos de aplicación en municipios, mancomunidades, comarcas, iniciativas de constitución de redes provinciales y autonómicas de municipios sostenibles y pactos de ciudades saludables y habitables. Según la naturaleza de los territorios, existen ejemplos demostrativos en espacios rurales, zonas rururbanas y en municipios con un marcado carácter urbano. Por ese motivo, creemos que España se sitúa a la vanguardia de nuestro ámbito geopolítico en cuanto al impulso del Programa 21 aunque aún queda mucho camino por recorrer como se demuestra en los balances de la situación que se han hecho 5 y 10 años después de la Cumbre de Río (Commission, 1997).

El proyecto de la Agenda 21 de la comarca Mancha Alta Conquense se centra en un territorio con una vocación rural clara. Se trata de una experiencia un tanto singular, por cuanto es el Grupo de Acción Local, que gestiona un programa de Desarrollo Rural, el que promueve la elaboración de la Agenda 21 a través de la Asociación para el Desarrollo Integral de los Municipios de la Mancha Alta Conquense. Esta experiencia plasma esa convergencia entre las políticas de desarrollo rural y de desarrollo sostenible en España y puede servir de referencia a otros programas similares.

Metodológicamente, existe un amplio consenso en cuanto al empleo de las auditorías medioambientales o ecoauditorías como herramienta objetiva de análisis y diagnóstico territorial y, por tanto, como documento de partida para la definición de los programas y líneas de acción estra-

Estudios Geográficos, LXIII, 248/249, 2002

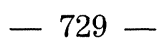


tégicos en el contexto del plan de acción local. Por otra parte, a pesar de las diferencias, existen numerosos puntos en común, entre las experiencias existentes, a la hora de estructurar las distintas fases que deben seguirse para la elaboración de las agendas 21 .

Por último, conviene refrendar la utilidad de los Sistemas de Información Geográfica como herramienta de apoyo imprescindible para el análisis y diagnóstico territorial y para proporcionar información geográfica, fundamental en el proceso de toma de decisiones. Igualmente, las encuestas de opinión a la población local son indispensables para conocer sus actitudes y su visión de futuro sobre el territorio.

\section{BIBLIOGRAFÍA}

Barragán, J. M., (2000): Agenda 21 Litoral de La Janda. Hacia un desarrollo sostenible, Cádiz, Universidad de Cádiz-Diputación de Cádiz, 64 pp.

CentRo de Estudios AMBIENTALES, (1998): Agenda 21: hacia un desarrollo sostenible, Vitoria, Ayuntamiento de Vitoria-Gastéiz, $81 \mathrm{pp}$.

CES, (2002): Informe sobre el documento de consulta para la Estrategia Española de Desarrollo Sostenible, Madrid, Consejo Económico y Social.

CoMmission (1997): Agenda 21: the first five years. European Community progress on the implementation of Agenda 21 1992-1997. Luxembourg. EU.

DeYNA, (1997): Agenda 21 Local. Guía para la elaboración de un modelo de desarrollo sostenible, Madrid, Fundación Desarrollo y Naturaleza, 59 pp.

Dva, (2002): Medi Ambient. Declaración de Xàtiva: red de municipios valencianos hacia la sostenibilidad, Diputación de Valencia, http://www.dva.gva.es/medioambiente/declara xativa.htm,(visto 21.11.02).

GenCAT, (2002): L'Agenda 21 de Catalunya, Generalitat de Catalunya, http://www.gencat.es/medioamb/sosten/a21-cat.htm,(visto 21.11.02).

GEOSCOPIO, (2002): «Los ayuntamientos solicitan una auditoría de la situación ambiental». Boletín Geoscopio, 23 de mayo de 2002, http://www.geoscopio.org/cgibin/geosco.../ntgeoscopio.cgi?\&idnoticias=7330\&acción=ve, (visto 28.05.02)

GobIERNo De NAvARra, (2002): Agenda Local 21 y Medio Ambiente Urbano, Gobierno de Navarra, http://www.cfnavarra.es/MedioAmbiente/Agenda/Agenda21/Ag21index.htm,(visto 21.11 .02$)$.

Gómez Orea, D. (2001): Ordenación Territorial, Madrid, Editorial Agrícola EspañolaMundi Prensa.

HewwitT, N., (1998): Guía europea para la planificación de las Agendas Locales 21, Vitoria, ICLEI-Gobierno Vasco.

JunTA DE ANDALUCÍA, (2000): Bases para la Agenda 21 Andalucía, Sevilla, Consejería de Gobernación.

J.CC. CaSTILla-La Mancha, (2002): Anuario estadístico de Castilla-La Mancha 2000. http://www.jccm.es/economía/anuario/frames/, (visto 5.03.02).

NACIONES UNIDAS, (1992): Conferencia Mundial de Naciones Unidas para el Desarrollo y el Medio Ambiente 1992 Rio de Janeiro, Nueva York, Naciones Unidas.

PRATS, F. y Miguélez, A., (1998): Lanzarote en la biosfera: una estrategia hacia el desarrollo sostenible de la isla: aplicación de la Agenda 21 Local a Lanzarote, Reserva de la

Estudios Geográficos, LXIII, 248/249, 2002

$$
-730-
$$


Biosfera: documento para el debate: el sistema insular, síntesis de la propuesta, Lanzarote, Cabildo Insular, 126 pp.

UCA (2002): Agenda 21 de la Costa Noroeste. Municipios gaditanos del Bajo Guadalquivir, Universidad de Cádiz, www.uca.es/grup-invest/coast/agenda21/default.html, (visto 21.11.02)

RESUMEN: Agenda 21 Local como instrumento de ordenación territorial: La Mancha Alta Conquense. El artículo pone de manifiesto la importancia de llevar a la práctica, desde la aplicación de conceptos y métodos geográficos, la recomendación del Programa 21 de impulsar el Desarrollo Sostenible en los territorios, mediante la implantación de la «Agenda 21 Local». Se revisan algunos de los documentos internacionales relacionados con las A21, así como algunas de las experiencias que sobre este tema se están desarrollando en España. Finalmente se expone la estructura y fases de la «Agenda 21 Comarcal de La Mancha Alta Conquense» que se ha puesto en marcha recientemente, así como los resultados que se esperan alcanzar.

Palabras Clave: Agenda 21 Local. Desarrollo Sostenible. Ordenación del Territorio. Comunidad Autónoma de Castilla-La Mancha.

ABSTRACT: The Local Agenda21 as a planning tool: «La Mancha Alta Conquense». The paper elaborates on the relevance of the of some Local Agenda21 policies implementation to stir up sustainable developments processes. It starts by reviewing different international documents and declarations, addressing some blunders in Spanish policies, which refer to the matter of concern, afterwards. Finally, the main chapters of the Agenda21 for «La Mancha Alta Conquense» are presented, and the results that it is supposed to attain.

Key Words: Local Agenda21. Sustainable Development. Land Planning. Castilla-La Mancha Autonomous Region, Spain. 